\title{
ESTUDIO DE LOS ABORDAJES DEL TERAPEUTA OCUPACIONAL EN LAS PERSONAS CON ATAXIA
}

\author{
STUDY OF OCCUPATIONAL THERAPIST'S APPROACHES IN PERSONS WITH ATAXIA
}

\section{Miguel Ángel Martínez Montesinos'; Marcos Martínez Pino².}

\begin{abstract}
RESUMEN
La ataxia es un tipo de trastorno del movimiento caracterizado por las alteraciones del equilibrio y de la coordinación, producida comúnmente por una disfunción cerebelosa. La mayoría de las personas que la padecen sufren secuelas que limitan o dificultan su autonomía para realizar las Actividades de la Vida Diaria (avD), afectando su desempeño ocupacional. El presente estudio tiene como principal objetivo explorar la bibliografía existente sobre las intervenciones de la Terapia Ocupacional (To) en las AVD de pacientes con ataxia.

La búsqueda bibliográfica se llevó a cabo en las bases de datos Pubmed, Índice Español de Ciencias Sociales y Humanidades (Isoc), Índice Médico Español (IME), Psicodoc, el portal de búsqueda de la Biblioteca Virtual en Salud (BvS) y Google Scholar. Finalmente, se analizaron un total de 6 artículos en lengua inglesa y española publicados desde el año 2000, que presentaban ensayos clínicos relacionados con la temática de búsqueda.
\end{abstract}

Existe escasa evidencia del beneficio de las intervenciones de la to en estos pacientes, aunque cabe destacar que las ganancias funcionales obtenidas a través de la intervención se mantienen en más de la mitad de los pacientes, siendo importante establecer un entrenamiento en las AVD para favorecer la independencia y autonomía el mayor tiempo posible. Todavía se necesitan más estudios científicos para generar mayor evidencia del rendimiento positivo de las intervenciones de la to en personas con ataxia.

DECS: Actividades de la Vida Diaria (AvD), Ataxia, Intervención, Terapia Ocupacional.

\section{PALABRAS CLAVE}

Actividades de la Vida Diaria (AvD), Ataxia, Intervención, Terapia Ocupacional.

1 Graduado en Terapia Ocupacional, Máster en Ciencias del Sistema Nervioso. Especialidad Neurorrehabilitación, Técnico Superior de

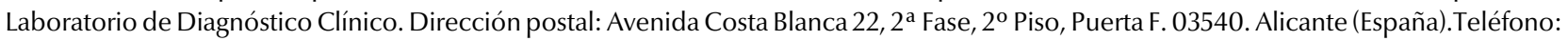
660829788. Correo: MiguelMMTO@hotmail.com

2 Máster en Salud Pública, Máster en Ciencias del Sistema Nervioso, Graduado en Terapia Ocupacional, Experto Universitario en Entrenamiento Personal. Dirección postal: Avenida de los Países Escandinavos 6, 6º F. 03540. Alicante (España). Teléfono: 637934366. Correo: marcospino75@gmail.com 


\section{ABSTRACT}

Ataxia is a type of movement disorder characterized by disturbances of balance and coordination, commonly produced by cerebellar dysfunction. The majority of people who suffer from it suffer from sequelae that limit or hinder their autonomy to perform the Activities of Daily Life (ADL), affecting their occupational performance. The present study has as main objective to explore the existing literature on Occupational Therapy (от) interventions in the ADL of patients with ataxia.

The bibliographic search was carried out in the databases Pubmed, Spanish Index of Social Sciences and Humanities (ISOC), Spanish Medical Index (IME), Psicodoc, the search portal of the Virtual Health Library (VHL) and Google Scholar. Finally, we analyzed a total of 6 articles in English and Spanish published since 2000, which presented clinical trials related to the search topic.

There is little evidence of the benefit of or interventions in these patients, although it should be noted that the functional gains obtained through the intervention are maintained in more than half of the patients, and it is important to establish a training in ADL for to promote independence and autonomy as long as possible. Further scientific studies are needed to generate further evidence of the positive performance of or interventions in people with ataxia.

MESH: Activity Daily Living, Ataxia, Intervention, Occupational Therapy.

\section{KEYWORDS}

Activity Daily Living, Ataxia, Intervention, Occupational Therapy.

Recibido 12/05/2016

Aceptado 27/09/2017 


\section{INTRODUCCIÓN}

La Terapia Ocupacional (то) es una disciplina sociosanitaria que promueve el uso terapéutico de la ocupación como parte del tratamiento, entendiendo como ocupación el conjunto de actividades del ser humano encaminadas al logro de objetivos. Cuando la salud se ve afectada, también lo hace la capacidad de realizar una ocupación y de participar en las Actividades de la Vida Diaria (AVD). El terapeuta ocupacional presta sus servicios a personas con algún problema que interfiere en el desempeño satisfactorio de su ocupación; llevar a cabo ocupaciones significativas para la persona es fundamental para su bienestar $(1,2)$.

La ataxia es un trastorno neurológico que abarca una serie de alteraciones de la coordinación motora voluntaria y del control postural, que lleva consigo una serie de dificultades que pueden afectar la calidad de vida del paciente y su vida laboral, pudiendo verse aumentada también la carga que soportan los cuidadores.

Muchos investigadores no la consideran una enfermedad en sí misma, sino más bien un síntoma propio de procesos degenerativos que causan discapacidad. El grado de progresión depende de factores genéticos, ambientales y personales; su origen, suele deberse a una disfunción cerebelosa, que se pone de manifiesto mediante temblores en diferentes partes del cuerpo durante la realización de movimientos voluntarios, así como mediante una clara incapacidad a la hora de controlar la amplitud de los mismos. Estos síntomas implican una serie de déficits en el desempeño de las AVD, afectando considerablemente a la calidad de vida. En este sentido, la To tendría cabida en el tratamiento de este tipo de pacientes al intervenir sobre los déficits, con el objetivo de lograr un desempeño ocupacional satisfactorio y facilitar el mantenimiento de las AVD $(3,4)$.

El fin de este trabajo tiene como principal propósito explorar la literatura científica existente sobre las intervenciones de la Terapia Ocupacional (то) en las AVD de pacientes con ataxia. Como objetivo secundario se planteó estudiar la eficiencia de las propias intervenciones de la тo en el proceso terapéutico de personas con ataxia.

\section{MÉTOdo}

Estrategia de búsqueda: Esta revisión bibliográfica se ha realizado a través de la página web de la Universidad Miguel Hernández (UMH), que facilita el acceso a diversas bases de datos relevantes del campo de las ciencias de la salud y las disciplinas sociosanitarias.

Las bases de datos utilizadas son las siguientes: Medline (a través de la plataforma de acceso gratuito Pubmed), Índice Español de Ciencias Sociales y Humanidades (ISOC), Índice Médico Español (IME), Psicodoc, el portal de búsqueda de la Biblioteca Virtual en Salud (Bvs) y, por último, en Google Scholar.

Tratamiento de la información: Las palabras clave utilizadas para realizar la búsqueda en castellano fueron: "Actividades de la Vida Diaria", "Ataxia", "Intervención" y "Terapia Ocupacional". Las palabras clave utilizadas en inglés fueron: "Activities of daily living", "Ataxia", "Intervention", "Occupational therapy".

La cobertura temporal se limitó a 15 años (20002015). Además de la búsqueda global, se realizó una búsqueda dirigida a localizar artículos publicados en español e inglés, y artículos de autores procedentes de instituciones españolas. La búsqueda bibliográfica se llevó a cabo en los meses de octubre y noviembre de 2015. Tras la descarga de los registros, fueron eliminados los duplicados y se revisaron todos los artículos para comprobar su pertinencia.

Selección de estudios: Los criterios de inclusión fueron: artículos con acceso gratuito al texto completo o con suscripción desde la UMH, publicados a partir del año 2000 en lengua española o inglesa. Los criterios de exclusión fueron: artículos que no se ajustasen al perfil de búsqueda. 


\section{Resultados}

Del total de los 40 artículos seleccionados en una primera exploración, finalmente, y tras varias cribas, se escogieron 6 artículos para este trabajo, ya que eran los artículos que cumplían con todos los criterios de selección.

Los resultados obtenidos después de realizar la búsqueda bibliográfica en las diferentes bases de datos utilizadas, han sido:

- En la base de datos Medline se obtienen 4 estudios científicos de los que son seleccionados 2 de acuerdo con los criterios de inclusión.

- El rastreo en la plataforma Google Scholar arroja un total de 32 resultados. Después de su revisión se extrajeron 2 artículos que se ajustaban a los criterios de búsqueda.

- De la plataforma de la Biblioteca Virtual en Salud (Bvs) se obtienen 11 artículos, de los que sólo se puede utilizar uno.

- La búsqueda en la base Psicodoc localizó un registro, pero no fue posible acceder al texto completo del mismo por falta de suscripción a la revista que lo contenía.

- La indagación realizada en el Índice Médico Español (IME) no obtuvo ningún resultado pertinente.

- Y por último, la base de datos Índice Español de Ciencias Sociales y Humanidades (ISOC) detectó otro trabajo que sí cumplía con los criterios establecidos.

A continuación, se presenta una síntesis de los seis artículos seleccionados:

En el estudio realizado por Sapiña González y colaboradores se expone la evaluación y el tratamiento de la Terapia Ocupacional (тo) de una paciente con Síndrome Opsoclono-Mioclono-Atáxico asociado a un neuroblastoma abdominal.

Para su correcta evaluación se realizó una valoración inicial, donde tras la revisión de la historia clínica, la entrevista familiar, la observación directa de la paciente, la recogida de información de otros profesionales como neurólogo, neuropediatra, fisioterapeuta, etc., se efectuaron las pruebas para su peritaje, que fueron: Perfil Sensorial de Winnie Dunn, Evaluación de Habilidades Motoras y de procesamiento (AMPS), y Pediatric Evaluation Disability Inventori (PEDI), para adquirir destrezas y realizar un análisis del desempeño ocupacional.

Los objetivos de la intervención fueron: mejorar la ejecución de las Actividades de la Vida Diaria (AVD), el procesamiento sensorial, la planificación motora, la percepción visual y, por último, las habilidades perceptivo-motoras.

Tras la reevaluación realizada después del tratamiento, y con las mismas escalas de la valoración inicial, la evolución fue positiva. Se pudo determinar que la paciente mejoró en el desempeño ocupacional de todas las áreas, aumentando su nivel de autonomía (5).

Gillen, en uno de los dos estudios suyos seleccionados, relata el caso clínico de un paciente con Esclerosis Múltiple que degenera en un Síndrome de Ataxia. En este caso, se ejecutó una evaluación inicial del paciente centrándose en las Actividades Básicas de la Vida Diaria (ABVD), ya que es el área más importante para la intervención. La Medida de Independencia Funcional (FIM) y el Índice de Barthel se manejaron como medidas estandarizadas del desempeño de las Actividades de la Vida Diaria. Tras obtener los resultados, se procedió a realizar una actuación desde la Terapia Ocupacional (то) con técnicas adaptativas que compensasen el efecto del trastorno del movimiento en el desempeño funcional en las Actividades Instrumentales de la Vida Diaria (AIVD) (preparación de la comida, uso del teléfono, etc.). En este caso, las intervenciones se centraron en la elaboración de estrategias para integrar el movimiento y el control disponible de la manera más eficaz y eficiente posible. Después de la intervención del terapeuta ocupacional, se reevaluó al paciente con las mismas escalas, siendo su resultado positivo, ya que fue capaz de participar de manera más independiente en las tareas realizadas (6).

Asimismo en Gillen, et al, se realizó un tratamiento en un paciente con Esclerosis Múltiple y Ataxia Severa, con el objetivo de fomentar la movilidad para favorecer su acceso a la comunidad. Para poder realizar la evaluación inicial se utilizó la escala de la Medida de Independencia Funcional (FIM). En este caso, el terapeuta ocupacional se centró en evaluar las Habilidades de la Vida Diaria y los factores que limitaban su ejecución. La actuación 
del terapeuta ocupacional se concentró en potenciar la estabilidad postural y mejorar el rendimiento de la movilidad. Tras la reevaluación, se pudo determinar que las intervenciones tuvieron resultados duraderos a pesar de la naturaleza crónica de la enfermedad. El paciente desarrolló sus habilidades, aumentando también su autonomía en las tareas significativas, fundamentalmente en su movilidad en la comunidad (7).

En el ensayo clínico de Miyai, y tras valorar inicialmente a los pacientes con la Escala para la Evaluación y Calificación de Ataxia (SARA) y la Medida de Independencia Funcional (FIM), se aplicaron intervenciones de la Terapia Ocupacional (To) en 42 pacientes con Enfermedades Degenerativas del Cerebelo. Los pacientes fueron reclutados de manera aleatoria en dos grupos, unos fueron asignados al grupo de intervención inmediata y otros al grupo control. Cada grupo recibió rehabilitación de то durante 4 semanas. Esta consistió en ejercitar el equilibrio, estimular el ejercicio de la marcha, la práctica de Actividades de la Vida Diaria (AVD). Después de participar cada uno de los grupos en el ensayo durante 4 semanas, se pudieron comparar los resultados. El primer grupo, el de intervención inmediata, mostró una recuperación funcional significativamente mayor que el grupo control, tanto en velocidad de la marcha, como en la realización de las AVD. Al parecer, cuanto antes se inicie la intervención del terapeuta ocupacional sobre los pacientes, los resultados que se obtengan serán superiores. En conclusión, el tratamiento precoz de la Terapia Ocupacional en pacientes con enfermedades degenerativas del cerebelo, puede ayudar a mantener las capacidades motoras para las Actividades de la Vida Diaria (AVD) (8).

En su estudio, Chisholm y coautores exponen que la participación del terapeuta ocupacional en las AVD de los pacientes consistía en entrenar el vestido, el aseo y la alimentación desde la bipedestación. La actuación del terapeuta ocupacional se centró en el entrenamiento de posturas dinámicas, trabajando el paciente al mismo tiempo la postura y la simetría del tronco. Este entrenamiento se realizó en 21 sesiones. Los objetivos de la intervención se centraron en mejorar la calidad de vida relacionada con la salud, también para mejorar el equilibrio, la propiocepción, y la coordinación media y fina. En este estudio, se utilizó para la evaluación inicial la Escala de Tinetti, Scale for the Assessment and Rating of Ataxia (SARA). Al concluir el tratamiento, se utilizaron las mismas escalas y parámetros en la evaluación final. Tras ser comparados los resultados con los obtenidos en los estudios iniciales se vio que el resultado de la evolución fue positivo en el paciente.

Como conclusión de este estudio, vemos que es necesario que las investigaciones se desarrollen de forma y manera que permitan que evolucione la rehabilitación en los pacientes atáxicos y que, al mismo tiempo, sirvan para tratar las afecciones que alteran la postura, la coordinación y el equilibrio (9).

En el artículo de revisión de Pérez y colaboradores se describe la incidencia del tratamiento de la Terapia Ocupacional en los pacientes con Ataxia de Fiedrich en un centro residencial para personas mayores. Tras la evaluación inicial, desarrollan y aplican un completo plan de intervención, exponiendo durante el desarrollo de todo el proceso cómo, tanto el paciente como el mismo plan, van siendo controlados por los terapeutas ocupacionales. Desde el departamento de Terapia Ocupacional (TO) se interviene en las Actividades de la Vida Diaria (AVD). Esta intervención se realiza en el aseo personal, alimentación, tareas domésticas y movilidad. El objetivo era conseguir el funcionamiento en las Actividades Básicas e Instrumentales de la Vida Diaria para obtener la mayor independencia posible. En este artículo se hace referencia sólo a un paciente de la institución, donde después de una exhaustiva exposición de la metodología utilizada para su diagnóstico, evaluación e intervención, finalmente no encontramos ninguna referencia a su evolución, no existiendo evidencia en el artículo de ninguna clase de resultado, ni positivo ni negativo (10).

\section{Discusión}

Desde sus inicios como profesión a principios del siglo $\mathrm{xx}$, los fundadores de la Terapia Ocupacional (то) han reconocido la importancia de poder establecer científicamente su eficacia para que la profesión fuera bien reconocida. Uno de ellos, George Barton, planteó que el tiempo y los estudios podrían ofrecer un modelo para la investigación en la to (11).

Tras la revisión bibliográfica realizada, vemos que se analizan y describen las principales dificultades en las Actividades de la Vida Diaria (AVD) que presentan las personas con ataxia. Estas dificultades conciernen sobre todo a la alimentación, el vestido, la higiene personal y la movilidad, generando en ellos diversos grados de de- 
pendencia. Las intervenciones de la to pueden conducir a una mejora de los problemas generados en la ataxia y de las funciones de la vida diaria en estos pacientes. Desde un punto de vista objetivo, se puede observar la utilidad de la To en el equipo multidisciplinar, como se señala en el artículo de Sapiña y colaboradores. Al realizar el trabajo, el paciente, logra una mejoría en la ejecución de las AVD a través del entrenamiento con el terapeuta ocupacional.

Tanto en el estudio elaborado por Gillen (2002) como en el realizado por Chisholm y coautores, se expone que, tras los análisis realizados, existe una relación directa entre la intervención de la to y la mejoría o estabilidad de los pacientes con ataxia, por lo que el tratamiento que se aplicará dependerá de la causa y del grado de afectación, mientras que el éxito del procedimiento dependerá del enfoque y de las aportaciones. Cabe señalar que estos estudios muestran una limitación incuestionable, basándose en un solo paciente, existiendo además el inconveniente de la poca evidencia que hay publicada sobre el cometido de la intervención de la to en la estabilidad del paciente con ataxia.

En el artículo de Gillen (2000), que presenta un análisis para comprobar si existe una mejora en la ejecución de las Actividades de la Vida Diaria (AVD) tras la intervención de la Terapia Ocupacional (To), se confirma que, a pesar de que los resultados finales no son estadísticamente significativos, éstos muestran una evolución general favorable en todos los ámbitos. Se debe tener en cuenta el error sistemático en el que se puede incurrir al hacer un muestreo, es un estudio con una representación pequeña. En este sentido, sería interesante seguir estudiando los posibles beneficios de este tipo de estrategias utilizando muestras mayores.

Por otra parte, en el estudio de Pérez y coautores, al parecer no existe una evidencia clara de los beneficios de la intervención de la To. Además, como en otros estudios anteriores, esta intervención también se realiza en un solo paciente. Por contra, en el estudio de Miyai se dispone de una población de intervención mayor, de 42 pacientes. Este último estudio es el que genera una mayor evidencia científica, por tratarse de un ensayo clínico aleatorizado, con casos y controles. Se declaran diversos beneficios del papel rehabilitador de la To en personas con ataxias, siendo los más destacados el mantenimiento y el desarrollo de las Actividades Básicas de la Vida Diaria. La mayoría de los artículos revisados, presentan la misma limitación, son poblaciones de tamaño reducido para poder obtener resultados más generales. En muchos casos las variables no eran lo suficientemente significativas para lograr extraer conclusiones. Como consecuencia, creo que sería oportuno realizar estudios con muestras más grandes y con mayor seguimiento, que permitan realizar este tipo de análisis.

Tras la realización de este trabajo de investigación, se plantea la necesidad de hacer más estudios longitudinales para medir y cuantificar los efectos beneficiosos de la intervención de la To en las AVD en los pacientes con ataxia. De esta manera, se podría obtener una mayor divulgación científica a través de las evidencias acreditadas de estas intervenciones. La falta de evidencia científica de la Terapia Ocupacional (то) en este campo puede deberse a la falta de financiación económica, la manera de valorar la eficacia y la eficiencia de los tratamientos de la To. Además, no se puede obviar la importancia de estas intervenciones para la mejora de la calidad de vida de los pacientes con ataxia. En la actualidad, los estudios publicados acerca de la Terapia Ocupacional en intervenciones de pacientes con ataxia son escasos, por lo que sería conveniente establecer diferentes líneas de intervención del terapeuta ocupacional en el proceso terapéutico de personas con ataxia para poder obtener una mayor evidencia de sus beneficios.

Las intervenciones del terapeuta ocupacional producen ciertos beneficios (aseo personal, alimentación, tareas domésticas y movilidad) que suelen depender del enfoque y del momento en que son Ilevadas a cabo. Es fundamental que se establezca un entrenamiento en las Actividades de la Vida Diaria, ya que son significativas para la mejoría de la independencia y la autonomía de estos pacientes y sus familias. El tratamiento dependerá de la causa y del grado de afectación. Cabe destacar que se necesitan más investigaciones contrastadas para poder formular las recomendaciones adecuadas en la rehabilitación del paciente atáxico. Además, y de este modo, se obtendría una mayor divulgación científica de los beneficios de estas intervenciones, al igual que favorecería la difusión a la población a través de los medios.

Por todo lo anteriormente expuesto es necesario que desde los propios terapeutas ocupacionales se investiguen sobre futuras líneas de intervención en los pacientes con ataxia. Se necesita más evidencia científica sobre la intervención de la Terapia Ocupacional para desarrollar tratamientos eficaces para estos pacientes. 


\begin{tabular}{|c|c|c|c|c|}
\hline 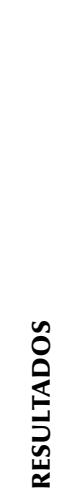 & 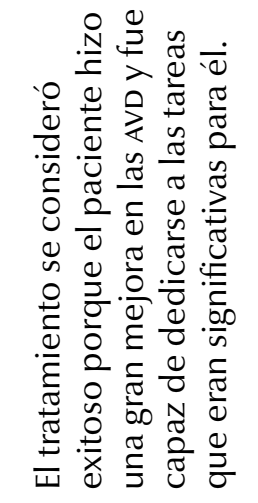 & 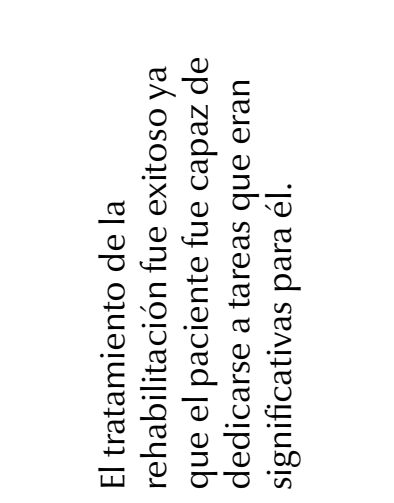 & 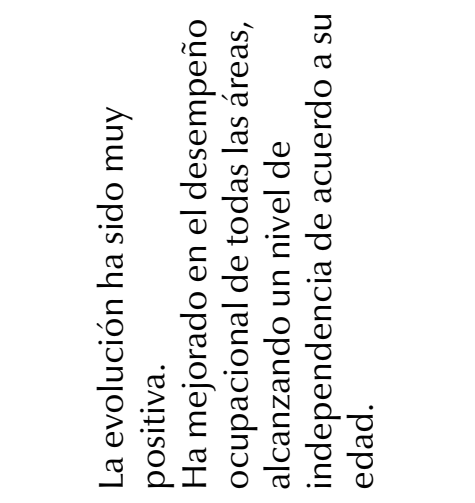 & 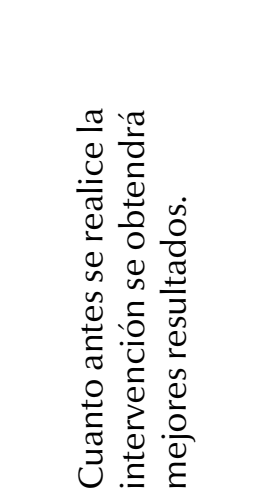 \\
\hline 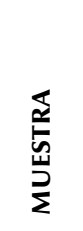 & 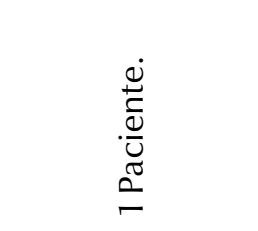 & 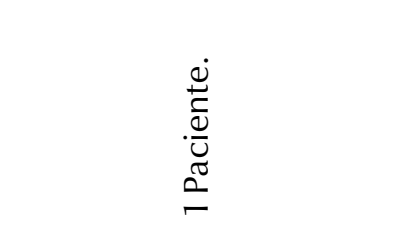 & 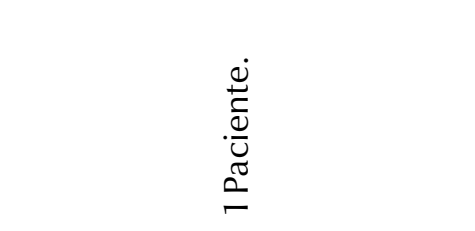 & 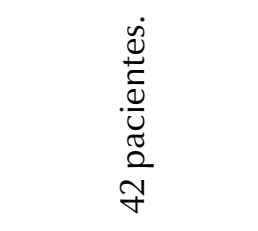 \\
\hline 号 & 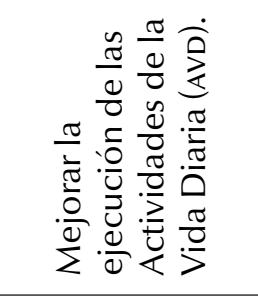 & 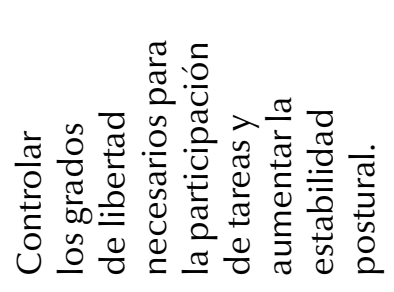 & 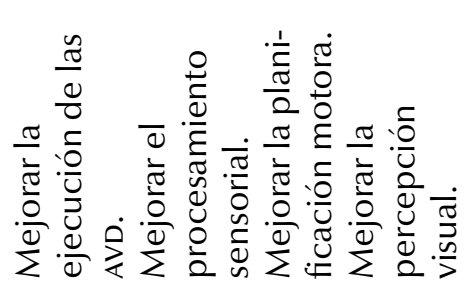 & 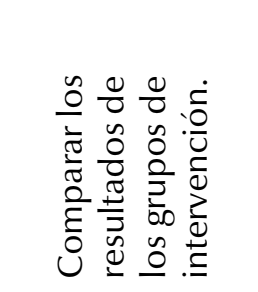 \\
\hline $\begin{array}{l}山 \\
0 \\
0 \\
0\end{array}$ & $\frac{\sqrt[0]{0}}{\stackrel{0}{\frac{n}{2}}}$ & $\frac{\sqrt[0]{0}}{\frac{0}{2}}$ & $\stackrel{8}{.}$ & : \\
\hline 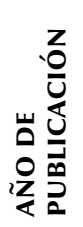 & $\begin{array}{l}\text { ஓ } \\
\text { ஓ }\end{array}$ & ஓ̊ & $\bar{\sigma}$ & 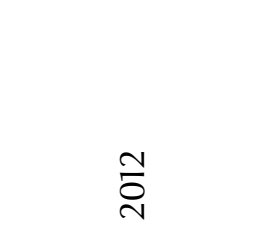 \\
\hline$\frac{2}{5}$ & $\begin{array}{l}\frac{\bar{d}}{\overline{\bar{U}}} \\
\frac{\bar{d}}{U}\end{array}$ & $\begin{array}{l}\frac{\bar{d}}{\overline{\bar{U}}} \\
\frac{\bar{d}}{U}\end{array}$ & 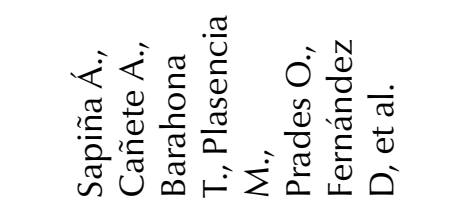 & $\frac{\dot{\bar{\pi}}}{\stackrel{\bar{\lambda}}{\Sigma}}$ \\
\hline$\stackrel{?}{?}$ & 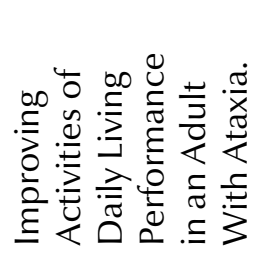 & 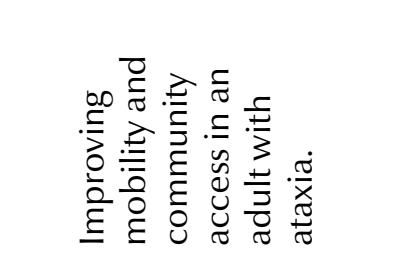 & 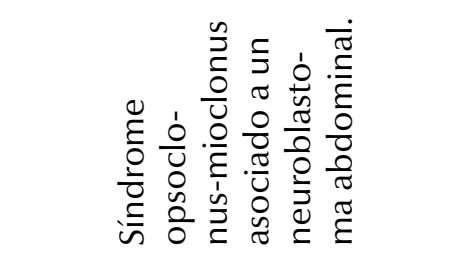 & 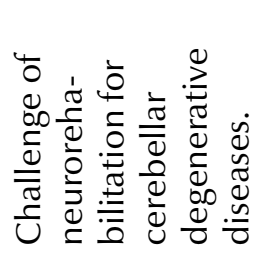 \\
\hline
\end{tabular}




\begin{tabular}{|c|c|c|}
\hline 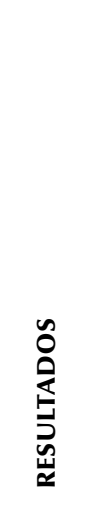 & 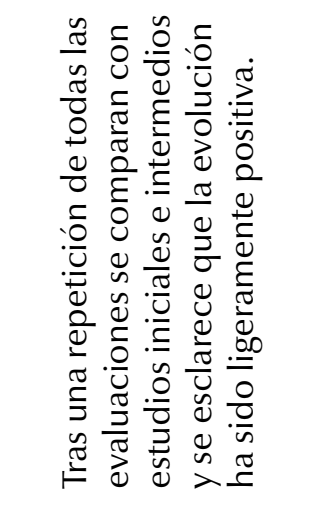 & 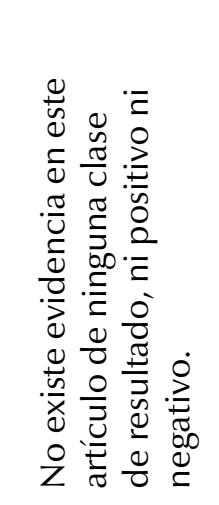 \\
\hline $\begin{array}{l}\frac{\pi}{5} \\
\frac{5}{2} \\
\frac{0}{2}\end{array}$ & 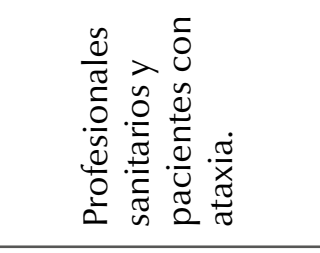 & 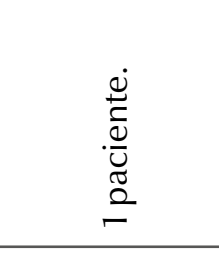 \\
\hline 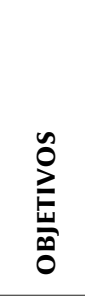 & 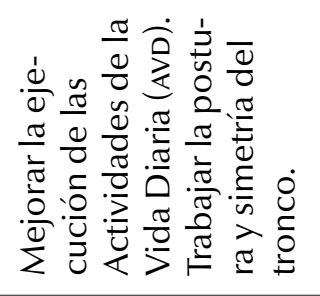 & 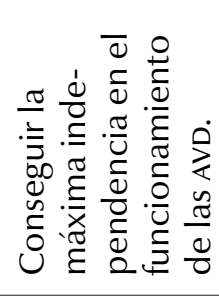 \\
\hline 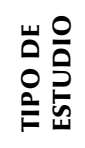 & 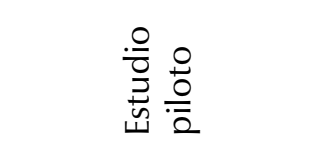 & 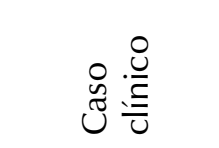 \\
\hline 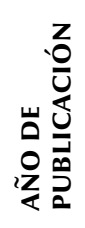 & 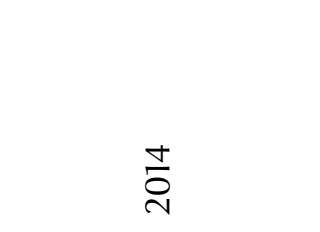 & 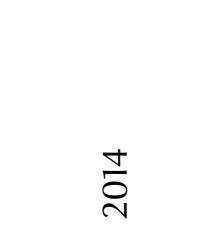 \\
\hline $\begin{array}{l}\frac{0}{0} \\
\frac{5}{2}\end{array}$ & 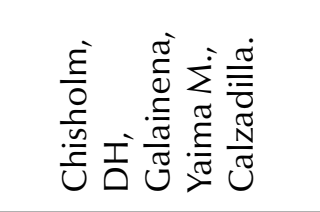 & 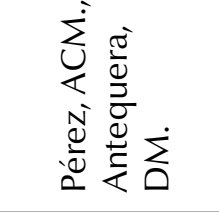 \\
\hline 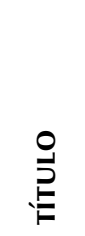 & 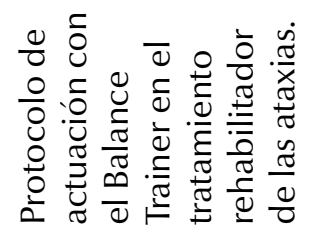 & 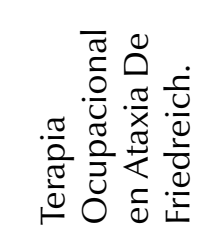 \\
\hline
\end{tabular}




\section{ReferenCiAs BibliográficAS}

De Heredia-Torres, M. P., \& Cuadrado-Pérez, M. (2002). Terapia ocupacional en neurología. Rev Neurol, 35(4), 366-372.

Kielhofner, G. (2006). Fundamentos conceptuales de la terapia ocupacional. $3^{\mathrm{a}}$ ed., pp 27-63). Buenos aires: Médica Panamericana.

Martínez, J. G., \& Nestal, C. T. (2006). Protocolos de Neurología. Boletín de Pediatría, 46(SUPL 1), 56-60.

Álvarez Melcón, Á. (2009). Ataxias hereditarias. REDUCA (Enfermería, Fisioterapia y Podología), 1(1)

Sapiña, Á., Cañete, A., Barahona, T., Plasencia, M., Moran, M., \& Prades, O. et al. (2012). Caso clínico: síndrome opsoclonus-mioclonus asociado a un neuroblastoma abdominal. Psicooncología, 8(2-3), 405-421. http://dx.doi.org/10.5209/rev_psic.2011.v8.n2-3.37889

Gillen, G. (2000). Improving Activities of Daily Living Performance in an adult with ataxia. American Journal Of Occupational Therapy, 54(1), 89-96. http://dx.doi.org/10.5014/ajot.54.1.89

Gillen, G. (2002). Improving Mobility and Community Access in an Adult With Ataxia. American Journal Of Occupational Therapy, 56(4), 462-466. http://dx.doi.org/10.5014/ajot.56.4.462

Miyai, I. (2011). Challenge of Neurorehabilitation for Cerebellar Degenerative Diseases. The Cerebellum, 11(2), 436-437. http://dx.doi.org/10.1007/s12311-011-0327-5

Cisneros Perdomo, V., Sánchez Castillo, Y., Hernández Chisholm, D., \& Calzadilla Galainena, Y. (2014). Protocolo de actuación con el Balance Trainer en el tratamiento rehabilitador de las ataxias. Revista Cubana De Medicina Física Y Rehabilitación, 2, 138-148. Retrieved from http://www.medigraphic.com/pdfs/revcubmedfisreah/cfr-2014/cfr142f.pdf

Pérez, A. C. M., \& Antequera, D. M. (2014). Terapia ocupacional en ataxia de Friedreich. Revista Electrónica De Terapia Ocupacional Galicia, TOG, (20), 15. Retrieved from http://www.revistatog.com/ num20/pdfs/caso1.pdf

Crepeau, E. B., Willard, H. S., Cohn, E. S., Spackman, C. S., \& Schell, B. A. B. (2005). Terapia ocupacional. 10 a ed. pp 5-13 Buenos aires: Médica Panamericana. 
